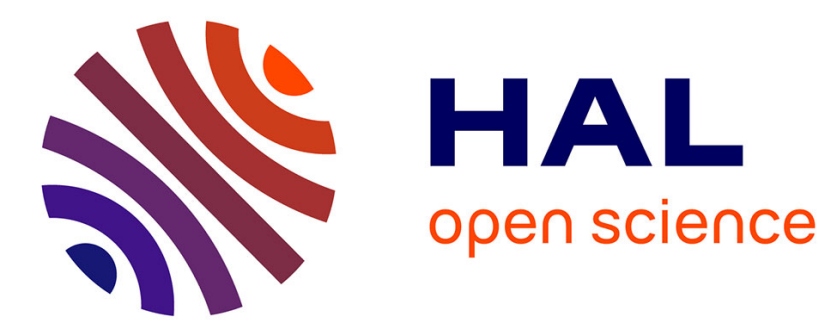

\title{
Radiative instability of an anticyclonic vortex in a stratified rotating fluid
}

\author{
Junho Park, Paul Billant
}

\section{To cite this version:}

Junho Park, Paul Billant. Radiative instability of an anticyclonic vortex in a stratified rotating fluid. Journal of Fluid Mechanics, 2012, 707 (september), pp.381-392. 10.1017/jfm.2012.286 . hal00996517

\section{HAL Id: hal-00996517 \\ https://hal-polytechnique.archives-ouvertes.fr/hal-00996517}

Submitted on 17 Jul 2014

HAL is a multi-disciplinary open access archive for the deposit and dissemination of scientific research documents, whether they are published or not. The documents may come from teaching and research institutions in France or abroad, or from public or private research centers.
L'archive ouverte pluridisciplinaire HAL, est destinée au dépôt et à la diffusion de documents scientifiques de niveau recherche, publiés ou non, émanant des établissements d'enseignement et de recherche français ou étrangers, des laboratoires publics ou privés. 


\title{
Radiative instability of an anticyclonic vortex in a stratified rotating fluid
}

\author{
Junho Park $\dagger$ and Paul Billant \\ LadHyX, CNRS, École Polytechnique, F-91128 Palaiseau CEDEX, France \\ (Received 28 February 2012; revised 30 April 2012; accepted 11 June 2012; \\ first published online 27 July 2012)
}

In strongly stratified fluids, an axisymmetric vertical columnar vortex is unstable because of a spontaneous radiation of internal waves. The growth rate of this radiative instability is strongly reduced in the presence of a cyclonic background rotation $f / 2$ and is smaller than the growth rate of the centrifugal instability for anticyclonic rotation, so it is generally expected to affect vortices in geophysical flows only if the Rossby number $R o=2 \Omega / f$ is large (where $\Omega$ is the angular velocity of the vortex). However, we show here that an anticyclonic Rankine vortex with low Rossby number in the range $-1 \leqslant R o<0$, which is centrifugally stable, is unstable to the radiative instability when the azimuthal wavenumber $|m|$ is larger than 2. Its growth rate for $R o=-1$ is comparable to the values reported in non-rotating stratified fluids. In the case of continuous vortex profiles, this new radiative instability is shown to occur if the potential vorticity of the base flow has a sufficiently steep radial profile. The most unstable azimuthal wavenumber is inversely proportional to the steepness of the vorticity jump. The properties and mechanism of the instability are explained by asymptotic analyses for large wavenumbers.

Key words: rotating flows, stratified flows, vortex instability

\section{Introduction}

When a vortex evolves in a fluid that can support waves, it can be unstable and spontaneously emit waves. Such radiative instability has been shown to occur in compressible fluids with the emission of acoustic waves (Broadbent \& Moore 1979), as well as shallow-water or rotating stratified fluids with the radiation of inertia-gravity waves (Ford 1994; Schecter \& Montgomery 2004, 2006; Schecter 2008; Billant \& Le Dizès 2009; Le Dizès \& Billant 2009). The instability comes from the coupling between the vortical waves sustained by the vortex and an outer wave field. Radiative instability has recently been thoroughly investigated in the case of a non-rotating stratified fluid. By means of a WKBJ analysis, Billant \& Le Dizès (2009) and Le Dizès \& Billant (2009) have shown that the instability can be understood as an over-reflection process. Riedinger, Le Dizès \& Meunier (2010) have investigated the effect of Reynolds and Froude numbers on the radiative instability of a Lamb-Oseen vortex. Riedinger, Le Dizès \& Meunier (2011) have also provided clear experimental evidence for the radiative instability in the case of flow around a rotating vertical cylinder in a stratified fluid.

The effect of planetary rotation has been analysed in the case of a cyclonic rotation (Schecter \& Montgomery 2004, 2006; Le Dizès \& Riedinger 2010). The growth

$\dagger$ Email address for correspondence: junho.park@ladhyx.polytechnique.fr 
rate of the radiative instability decreases with the Rossby number and becomes exponentially small in the quasi-geostrophic limit (Vanneste \& Yavneh 2004, 2007). In the presence of anticyclonic rotation, the centrifugal instability, which is stronger, occurs when $R o<-1$. Hence, it is generally concluded that the radiative instability will not affect real vortices in the oceans and atmosphere if the Rossby number is smaller than unity (Schecter \& Montgomery 2004; Le Dizès \& Riedinger 2010). However, we show in this paper that the radiative instability can occur on a columnar anticyclonic vortex with a non-negligible growth rate when $-1<R o<0$, i.e. in the centrifugally stable regime. Interestingly, this range of Rossby numbers corresponds to many large-scale vortices observed in the oceans such as, for example, the 'Meddies' (Ménesguen et al. 2009).

The paper is organized as follows. The problem is formulated in $\S 2$. In $\S 3$, we explain why radiative instability can be expected to occur in the range $-1 \leqslant R o<0$ above a critical azimuthal wavenumber. In $\S \S 4$ and 5 , the stability of the Rankine vortex and the smoothed Rankine vortex are investigated.

\section{Problem formulation}

We consider an axisymmetric vortex with velocity components $\left(0, U_{\theta}(r), 0\right)$ in a cylindrical coordinate system $(r, \theta, z)$ which is rotating about the vertical axis at angular velocity $\Omega_{b}=f / 2$, where $f$ is the Coriolis parameter. The fluid is assumed to be inviscid and stably stratified with a constant Brunt-Väisälä frequency $N$. We subject this vortex to infinitesimally small three-dimensional perturbations of velocity $\tilde{\boldsymbol{u}}=\left(\tilde{u}_{r}, \tilde{u}_{\theta}, \tilde{u}_{z}\right)$, pressure $\tilde{p}$ and density $\tilde{\rho}$, written in the form $(\tilde{\boldsymbol{u}}, \tilde{p}, \tilde{\rho})=$ $(\boldsymbol{u}(r), p(r), \rho(r)) \mathrm{e}^{-\mathrm{i} \omega t+\mathrm{i} k z+\mathrm{i} m \theta}+$ c.c., where $\omega$ is the complex frequency, $m$ the azimuthal wavenumber and $k$ the vertical wavenumber. Under the Boussinesq approximation, the linearized equations of momentum, density conservation and continuity for the perturbations can be reduced to a single equation for the pressure perturbation $p$ :

$$
\frac{\mathrm{d}^{2} p}{\mathrm{~d} r^{2}}+\left(\frac{1}{r}-\frac{\Delta^{\prime}}{\Delta}\right) \frac{\mathrm{d} p}{\mathrm{~d} r}+\left[-\frac{k^{2}}{N^{2}-s^{2}} \Delta-\frac{m^{2}}{r^{2}}+\frac{m \Delta}{r s}\left(\frac{f+2 U_{\theta} / r}{\Delta}\right)^{\prime}\right] p(r)=0,
$$

where $\Delta(r)=(f+\zeta)\left(f+\left(2 U_{\theta} / r\right)\right)-s^{2}, \zeta=(1 / r)(\mathrm{d} / \mathrm{d} r)\left(r U_{\theta}\right)$ is the axial vorticity of the vortex, $s=-\omega+m U_{\theta} / r$ is the Doppler-shifted frequency, and the prime denotes differentiation with respect to $r$ (Smyth \& McWilliams 1998). Due to the symmetry $\omega(k, m)=\omega(-k, m)=-\omega^{*}(-k,-m)$, we consider hereafter only positive $k$ and $m$. Moreover, we assume that the fluid is strongly stratified, so the hydrostatic approximation can be applied: $N \gg|s|$ (i.e. $N^{2}-s^{2} \approx N^{2}$ ). This implies that (2.1) depends on $k$ and $N$ only through the rescaled vertical wavenumber $\tilde{k}=k / N$ owing to the self-similarity of strongly stratified fluids (Billant \& Chomaz 2001). This assumption also implies that there is no singularity where $|s|=N$.

In the following, we first consider as the basic state the Rankine vortex, $U_{\theta}(r)=\Omega r$ for $r<R$ and $U_{\theta}(r)=\Omega R^{2} / r$ for $r>R$, where $\Omega$ is constant and $R$ is the radius of the vortex core. A continuous smoothed Rankine vortex will be considered next in $\S 5$. The boundary conditions are that the perturbations are non-singular at the vortex centre $r=0$ and decay exponentially or radiate energy outward as $r \rightarrow \infty$. In the case of the Rankine vortex, we also apply the kinematic and the dynamic conditions at the boundary of the vortex core $r=R: u_{r_{\text {in }}}(R)=u_{r_{\text {out }}}(R)$ and $p_{\text {in }}(R)=p_{\text {out }}(R)$, respectively. The subscripts ' $i n$ ' and ' $o u t$ ' denote the solution for $r<R$ and $r>R$, respectively. By using the relation $u_{r} \sim-\mathrm{i}\left\{s p^{\prime}+(m / r)\left(f+\left(2 U_{\theta} / r\right)\right) p\right\} / \Delta$ derived from the horizontal 
momentum equations, these boundary conditions give the dispersion relation in terms of $p$ :

$$
\frac{p_{\text {out }}^{\prime}(R)}{p_{\text {out }}(R)}=\frac{\Delta_{\text {out }}(R)}{\Delta_{\text {in }}(R)} \frac{p_{\text {in }}^{\prime}(R)}{p_{\text {in }}(R)}-\frac{m(f+2 \Omega)}{R s(R)}\left[1-\frac{\Delta_{\text {out }}(R)}{\Delta_{\text {in }}(R)}\right] .
$$

Note that $\Delta_{\text {in }}(R) \neq \Delta_{\text {out }}(R)$, since there is a discontinuity of the axial vorticity $\zeta(r)$ for the Rankine vortex. The non-singular solution of (2.1) inside the vortex core $(r<R)$ is

$$
p_{i n}(r) \sim \mathrm{J}_{m}\left(\tilde{k} r \sqrt{(-\omega+m \Omega)^{2}-(f+2 \Omega)^{2}}\right),
$$

where $\mathbf{J}_{m}$ is the Bessel function of the first kind of order $m$. When $\omega$ is real, the function $\mathrm{J}_{m}$ has a wave behaviour when $|-\omega+m \Omega|>|f+2 \Omega|$ and increases exponentially when $|-\omega+m \Omega|<|f+2 \Omega|$. Outside the vortex core $(r>R)$, (2.1) needs to be integrated numerically. The integration is performed inward, starting far outside the vortex core $r \gg R$ from the asymptotic solution, which satisfies the boundary condition for $r \rightarrow \infty$,

$$
p_{\text {out }}(r) \sim \mathrm{H}_{m}^{(1)}\left(\tilde{k} r \sqrt{\omega^{2}-f^{2}}\right),
$$

where $\mathrm{H}_{m}^{(1)}$ is the Hankel function of the first kind of order $m$. For real $\omega, \mathrm{H}_{m}^{(1)}$ behaves like an outgoing wave when $|\omega|>|f|$ and decreases exponentially with $r$ when $|\omega|<|f|$. Starting from an initial estimate for $\omega$, the secant method is used to find the value of $\omega$ which satisfies the dispersion relation (2.2).

\section{Preliminary discussion}

In the present paper, we shall consider anticyclonic background rotation in the range $-1 \leqslant R o<0$, where $R o=2 \Omega / f$ is the Rossby number. In this range, the Rankine vortex is stable with respect to the centrifugal instability since the Rayleigh discriminant $\phi=(f+\zeta)\left(f+2 U_{\theta} / r\right)$ is positive for all radii (Kloosterziel \& van Heijst 1991). However, we shall show that radiative instability can occur. Its existence can be anticipated thanks to a WKBJ analysis for large rescaled axial wavenumber $\tilde{k}=k / N$ following Le Dizès \& Lacaze (2005), Billant \& Le Dizès (2009) and Le Dizès \& Billant (2009). For large $\tilde{k}$, the WKBJ approximation of the solution of (2.1) is

$$
p(r)=A \frac{(-\Delta)^{1 / 4}}{r^{1 / 2}} \mathrm{e}^{\mathrm{i} \tilde{k} \int_{r_{t}}^{r} \sqrt{-\Delta(t)} \mathrm{d} t}+B \frac{(-\Delta)^{1 / 4}}{r^{1 / 2}} \mathrm{e}^{-\mathrm{i} \tilde{k} \int_{r_{t}}^{r} \sqrt{-\Delta(t)} \mathrm{d} t},
$$

where $(A, B)$ are constants (Bender \& Orszag 1978). This approximation is valid everywhere except in the neighbourhood of the so-called turning point $r_{t}$, where $\Delta\left(r_{t}\right)=0$. From (3.1), we see that the WKBJ approximation is wavelike if $\Delta<0$ while it is exponentially decreasing or growing if $\Delta>0$. The sign of $\Delta$ can be easily determined by plotting the epicyclic frequencies $\omega_{ \pm}(r)=m U_{\theta} / r \pm$ $\sqrt{(f+\zeta)\left(f+2 U_{\theta} / r\right)}$ (Le Dizès \& Lacaze 2005). When the frequency $\omega$ lies in the interval $\omega_{-}<\omega<\omega_{+}, \Delta$ is positive; otherwise $\Delta$ is negative. Le Dizès \& Billant (2009) have shown that a condition for the existence of radiative instability is the presence of two intervals where $\Delta$ is negative, i.e. where the WKBJ approximation is wavelike, one of which extends towards infinity. These two regions should be separated by a third region, where $\Delta$ is positive and which contains a critical radius $r_{c}$ where $s\left(r_{c}\right)=0$, i.e. where the azimuthal phase velocity $\omega_{c} / m$ is equal to the angular velocity $U_{\theta}\left(r_{c}\right) / r_{c}$. When these conditions are fulfilled, the group velocity reverses 

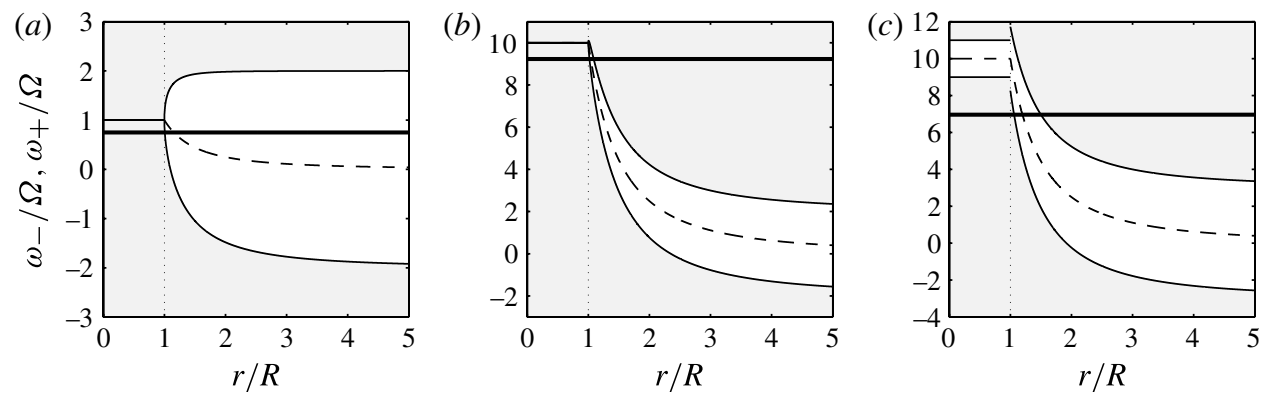

FIGURE 1. Epicyclic frequencies $\omega_{-}$and $\omega_{+}$(solid lines) and critical frequency $\omega_{c}$ (dashed lines) as a function of $r$ for $(a)(m, R o)=(1,-1),(b)(m, R o)=(10,-1)$, and (c) $(m, R o)=(10,-2 / 3)$. The regions where the WKBJ approximations are wavelike (i.e. $\Delta<0)$ are shaded. Dotted lines represent the boundary of the vortex core. Thick solid lines show examples of the frequency $\omega_{r}$.

at $r_{c}$, so an incident wave propagating from the inner region where $\Delta<0$ toward the outer one is over-reflected at the critical radius, leading to unstable eigenmodes.

Figure 1 shows the frequencies $\omega_{ \pm}$and $\omega_{c}$ for different examples of $m$ and $R o$. The regions where $\Delta$ is negative are shaded. For $m=1$ and $R o=-1$ (figure $1 a$ ), we see that there is never co-existence of two regions where $\Delta<0$ for a given frequency $\omega$. This remains the case for any Rossby number in the range $[-1,0]$, so radiative instability is not expected to exist for $m=1$. In contrast, for $m=10$ and $R o=-1$ (figure $1 b$ ) and $R o=-2 / 3$ (figure $1 c$ ), there exist two wave regions $(\Delta<0)$ separated by a region where $\Delta>0$ and which contains the critical frequency $\omega_{c}$ (dashed lines) for the examples of frequency indicated by a bold line. For arbitrary $m$ and $R o$, it is easy to deduce that such a radiative configuration can exist only if $\omega_{+}(\infty)<\omega<\max \left(\omega_{-}\right)$, because $\omega_{-}$always decreases monotonically with $r$ for the Rankine vortex. The first inequality $\omega_{+}(\infty)<\omega$ ensures that there exists a radiating wave at infinity whereas the second inequality $\omega<\max \left(\omega_{-}\right)$implies that there are at least two wave regions. Using the expressions of the epicyclic frequencies $\omega_{ \pm}$for the Rankine vortex, this gives

$$
-\frac{2}{R o}<\frac{\omega}{\Omega}<m+\frac{2}{R o}+2 .
$$

Hence, a condition for the existence of radiative instability is $\omega_{+}(\infty)<\max \left(\omega_{-}\right)$, i.e. $R o<-4 /(m+2)$. Therefore, radiative instability should exist for large axial wavenumber in the range $-1 \leqslant R o<0$ only if $m>2$. We shall now integrate (2.1) numerically in order to check these predictions. The detailed derivation of the complete WKBJ approximations in the different regions is postponed to the Appendix.

\section{Stability of the Rankine vortex}

\subsection{Numerical results for $\mathrm{Ro}=-1$}

In figure 2, we show the frequency and growth rate for $m=1$ and $m=10$ at $R o=-1$. These two azimuthal wavenumbers are typical of the two different behaviours that can be encountered when $m$ is varied. As anticipated in $\S 3$, the azimuthal wavenumber $m=1$ is neutral, whereas for $m=10$ the growth rate is positive (figure $2 b$ ). For both azimuthal wavenumbers, there is an infinite number of branches which differ by the number of zeros of the eigenfunction inside the vortex core. The first branch 

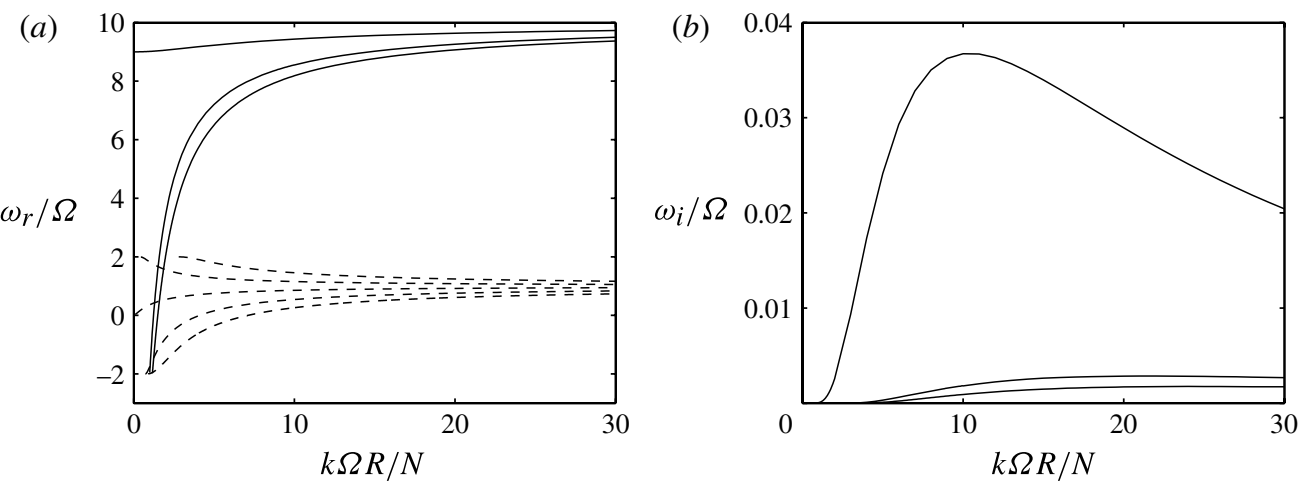

FIGURE 2. (a) Frequency $\omega_{r}$ and $(b)$ growth rate $\omega_{i}$ as a function of the rescaled vertical wavenumber $k \Omega R / N$ at $R o=-1$. Dashed lines and solid lines are numerical results for $m=1$ and $m=10$, respectively. Only the first three branches are shown.
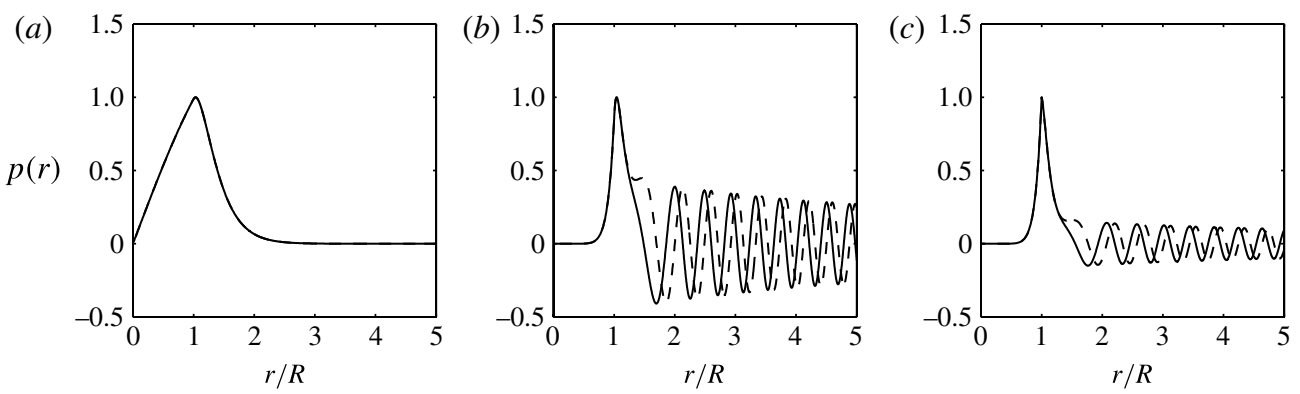

FIGURE 3. Eigenfunctions $p(r)$ for $(a)(m, R o)=(1,-1),(b)(m, R o)=(10,-1)$, and (c) $(m, R o)=(10,-2 / 3)$ for the first branch at $k R \Omega / N=2$. The solid and dashed lines are the real part and imaginary part, respectively.

starts at $k=0$ from the two-dimensional dispersion relation $\omega=(m-1) \Omega$, and tends to $m \Omega$ as $k$ increases regardless of $R o$ and $m$. In contrast, the next branches start at a finite axial wavenumber $k>0$ and $\omega / \Omega=\omega_{-}(\infty) / \Omega=2 / R o$ and tend to $\omega / \Omega=\omega_{-}(0) / \Omega=m+(2 / R o+2)$ as $k \rightarrow \infty$. This frequency range corresponds to the configurations for which there exist a confined inner wave region. For $m=1$, there is another family of branches which start from $\omega / \Omega=\omega_{+}(\infty) / \Omega=-2 / R o$ and tend to $\omega / \Omega=\omega_{+}(0) / \Omega=m-(2 / R o+2)$ as $k \rightarrow \infty$. This family of branches exists only if $m-(2 / R o+2)<-2 / R o$ (i.e. only if $m<2)$. As seen in figure $2(b)$, the first branch for $m=10$ is the most unstable and the growth rate of the next branches is much smaller, and only positive when $\omega_{r}$ is in the range (3.2), i.e. only when there exist two wave regions (figure $1 b$ ).

Some examples of pressure eigenfunctions for the first branch for $m=1$ and $m=10$ for $R o=-1$ are displayed in figures $3(a)$ and $3(b)$. A radiating inertia-gravity wave train is clearly visible outside the vortex core for $m=10$ (figure $3 b$ ) in contrast to $m=1$ (figure $3 a$ ).

\subsection{Variation with the azimuthal wavenumber}

Figure 4 shows the frequency $\omega_{r}$ and growth rate $\omega_{i}$ of the first branch (i.e. the most unstable branch) for several azimuthal wavenumbers from $m=1$ to $m=50$ 

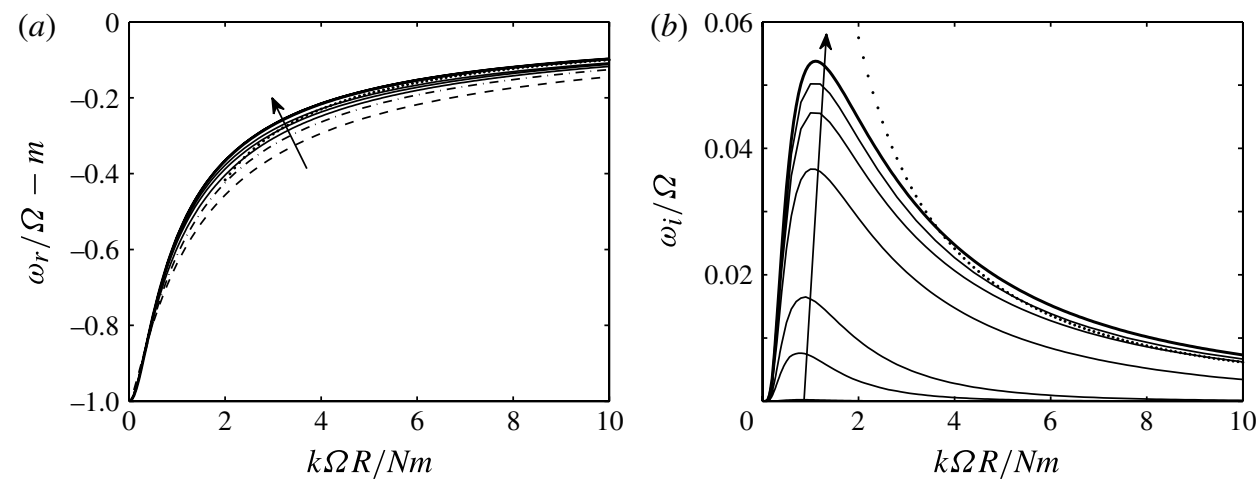

FIgURE 4. (a) Rescaled frequency $\left(\omega_{r} / \Omega-m\right)$ and $(b)$ growth rate $\omega_{i}$ as a function of rescaled vertical wavenumber $k \Omega R /(\mathrm{Nm})$ at $R o=-1$. The dashed line, dot-dashed line and solid lines are numerical results for $m=1, m=2$ and $m=[3,4,5,10,20,50]$. The arrows indicate an increase of $m$. The bold and dotted lines are asymptotic results for large $m(4.2)$ and for both large $\tilde{k}$ and $m$ (A 6 ) for $m=20$, respectively.

for $R o=-1$. Following Le Dizès \& Riedinger (2010), the frequency and vertical wavenumber have been rescaled as $\omega_{r} / \Omega-m$ and $k R \Omega /(N m)$. With these scalings, the frequency of every azimuthal wavenumber collapses approximately on the same curve (figure $4 a$ ). As seen in figure $4(b)$, the growth rate is positive only if $m \geqslant 3$, as predicted in $\S 3$, and increases monotonically with $m$. Le Dizès \& Riedinger (2010) (see also Candelier, Le Dizès \& Millet 2012) have shown that the limit $m \rightarrow \infty$ can be analysed asymptotically by introducing the rescaled variables: $\bar{r}=m(r / R-1)$, $\omega_{0}=\omega / \Omega-m, k_{1}=k R \Omega /(N m)$ where $\left(\bar{r}, \omega_{0}, k_{1}\right)$ and $R o$ are assumed to be of order one. For large $m,(2.1)$ then becomes at leading order

$$
\frac{\mathrm{d}^{2} p}{\mathrm{~d} \bar{r}^{2}}-\frac{1}{\bar{\Delta}} \frac{\mathrm{d} \bar{\Delta}}{\mathrm{d} \bar{r}} \frac{\mathrm{d} p}{\mathrm{~d} \bar{r}}+\left[-k_{1}^{2} \bar{\Delta}-1-8\left(\frac{1+R o}{R o \bar{\Delta}}\right)\right] p=O\left(\frac{1}{m}\right),
$$

where $\bar{\Delta}(\bar{r})=4(1+R o) / R o^{2}-\left(\omega_{0}+2 \bar{r}\right)^{2}$. This equation is valid around $\bar{r} \sim O(1)$ (i.e. $r \sim R)$ and it has to be solved numerically except for the particular value $R o=-1$, where an analytical solution can be found:

$$
p(\bar{r})=C \mathrm{e}^{-\mathrm{i} \bar{z}}[M(a, b, 2 \mathrm{i} \bar{z})+\gamma U(a, b, 2 \mathrm{i} \bar{z})],
$$

where $C$ is a constant, $\bar{z}=k_{1}\left(\omega_{0}+2 \bar{r}\right)^{2} / 4, a=-1 / 4-\mathrm{i} /\left(8 k_{1}\right), b=-1 / 2$, and $M$ and $U$ denote the Kummer functions (Abramowitz \& Stegun 1965). If one imposes $\gamma=-\Gamma(-1 / 2) \exp ^{\mathrm{i} \pi a} / \Gamma(-a-1 / 2)$, where $\Gamma$ is the Gamma function, then (4.2) matches the outgoing wave (2.4) as $\bar{r} \rightarrow \infty$.

The results obtained by using the asymptotic solution (4.2) in the dispersion relation (2.2) are shown by bold lines in figure 4. We see that the numerical results tend to these asymptotic results as $m$ increases. The maximum growth rate in the limit $m \rightarrow \infty$ is $\omega_{i} \approx 0.053 \Omega$ and is reached for $k R \Omega /(N m) \approx 1$. It is worth pointing out that this growth rate is of the same order of magnitude as the values computed in nonrotating stratified fluids (Billant \& Le Dizès 2009) and for the flow around a rotating cylinder in strongly stratified fluids in the range Ro $>0$ (Le Dizès \& Riedinger 2010; Riedinger et al. 2011). Note, however, that the first branch is absent in the case of the flow around a rotating cylinder because of the different boundary condition on the cylinder. 

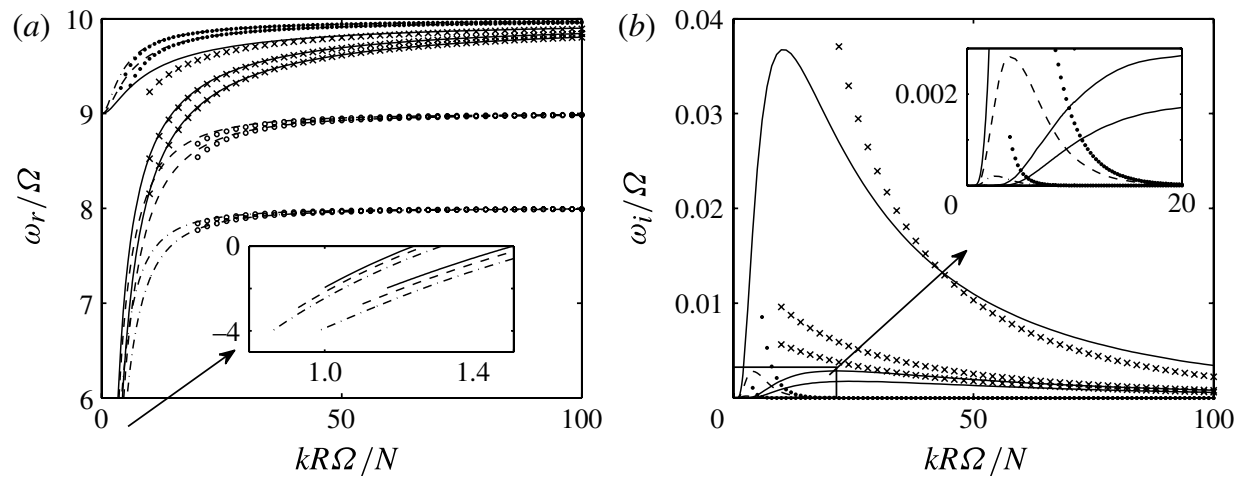

FIGURE 5. (a) Frequency $\omega_{r}$ and $(b)$ growth rate of the first three branches for $m=10$ for different Rossby numbers: solid lines, $R o=-1$; dashed lines, $R o=-2 / 3$; dot-dashed lines, $R o=-1 / 2$. Symbols represent the WKBJ predictions for large $\tilde{k}(\bullet$ (A 3), ○ (A 4)) and for both large $\tilde{k}$ and $m(\times(\mathrm{A} 6))$. The inset in (a) shows the region around $\omega / \Omega=2 / R o$ and the inset in $(b)$ shows the region around $k=0$. For $R o=-2 / 3$ and $R o=-1 / 2$, only the growth rate of the first branch is displayed because the growth rate of the next branches is too small.

\subsection{Effect of the Rossby number}

Figure 5 shows the frequency and the growth rate of the first three branches for three different Rossby numbers, $R o=-1,-2 / 3$ and $-1 / 2$, for the typical azimuthal wavenumber $m=10$. In figure $5(a)$, we can see that the frequency of the first branch lies in the range $[(m-1) \Omega, m \Omega]$ independently of $R o$. This is observed for all the azimuthal wavenumbers. In contrast, the frequency range of the next branches depends on $R o$, and corresponds when $m \geqslant 3$ to the range $\omega_{-}(\infty)<\omega<\omega_{-}(0)$, for which a confined inner wave region exists, as discussed in $\S 4.1$. The frequency predicted by the WKBJ analyses that are performed in the Appendix are plotted by symbols in figure 5(a). The asymptotic and numerical frequencies are in good agreement for large vertical wavenumber $\tilde{k}$. The growth rate predicted by the WKBJ analyses is plotted with the same symbols in figure $5(b)$. It is in good agreement with the numerical growth rate only for large axial wavenumber.

The maximum growth rate as a function of $R o$ is plotted in figure 6 . We can see that it decreases quickly when $R o$ is increased from $R o=-1$ and becomes very small when $R o \gtrsim-0.4$. The maximum growth rate is always attained by the first branch regardless of the Rossby number. Since the frequency of the first branch is always in the range $(m-1) \Omega<\omega<m \Omega$, we can deduce by considering the epicyclic frequencies $\omega_{ \pm}$that there is only a single outer wave region and no inner wave region for the first branch when $-2 / 3 \leqslant R o<-2 / m$ for any $m$ (see the example in figure $1 c$ ). Quite strikingly, the growth rate remains positive for $R o \geqslant-2 / 3$ and there is a strong wave emission (figure 3c). As explained in the Appendix, there is actually a second wave region concealed in the vorticity jump at $r=R$. When $R o>-2 / m$, there is no outer wave region and the growth rate is purely zero.

\section{Stability of the smoothed Rankine vortex}

We now investigate the stability of a more realistic vortex profile with a continuous vorticity profile. More specifically, we consider the smoothed Rankine 


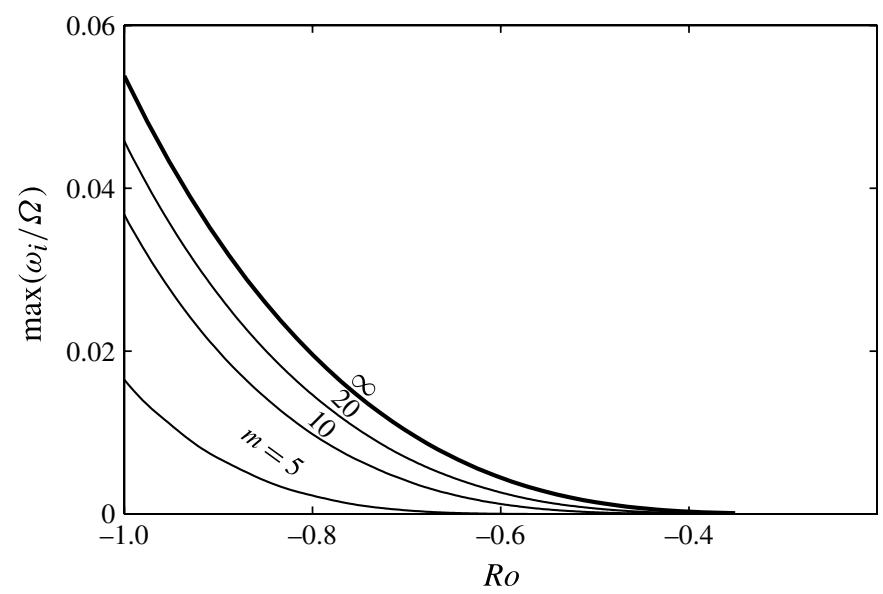

FIgURE 6. Maximum growth rate $\max \left(\omega_{i}\right)$ of the first branch as a function of $R o$ for different $m$. The bold line shows the limit $m=\infty$ computed from (4.1).
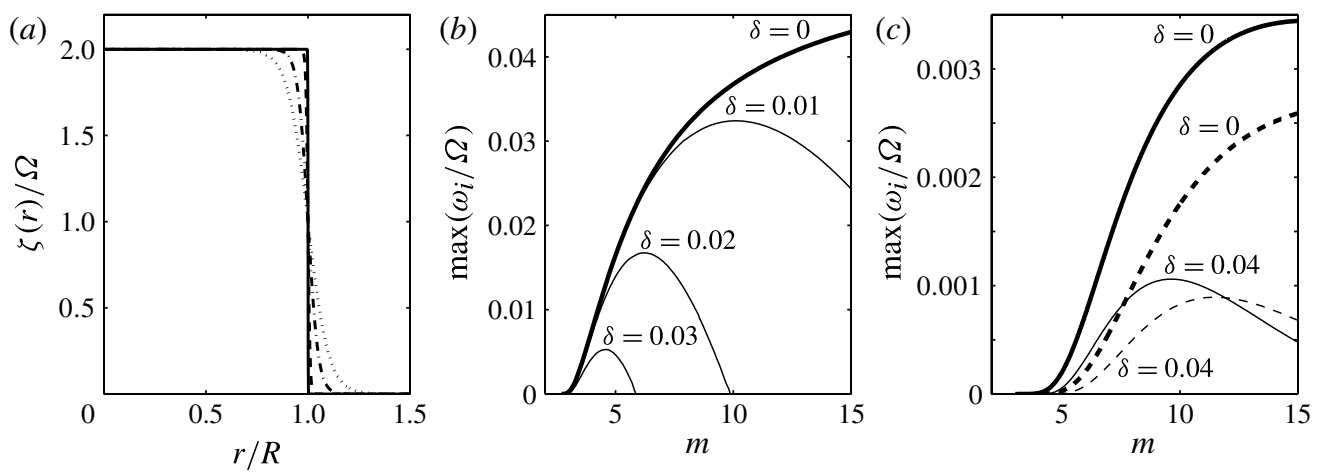

FIGURE 7. (a) Vertical vorticity of the smoothed Rankine vortex (5.1) for $\delta=0.001$ (solid lines), $\delta=0.01$ (dashed lines), $\delta=0.05$ (dot-dashed lines) and $\delta=0.1$ (dotted lines). $(b)$ Maximum growth rate $\max \left(\omega_{i}\right)$ of the first branch as a function of $m$ for different values of $\delta$ for $R o=-1$. (c) Maximum growth rate $\max \left(\omega_{i}\right)$ of the second branch (solid lines) and the third branch (dashed lines) as a function of $m$ for $\delta=0$ and $\delta=0.04$ for $R o=-1$. The bold lines in $(b, c)$ represent the limit $\delta=0$ (i.e. the Rankine vortex).

vortex introduced by Schecter \& Montgomery (2004) with axial vorticity

$$
\zeta(r)=\Omega\left[1-\tanh \left(\frac{r / R-1}{\delta}\right)\right],
$$

where $\delta$ is a smoothness parameter. As illustrated in figure 7(a), the vorticity jump becomes smoother as $\delta$ increases from zero. For this profile, a classical shooting method is used to solve (2.1) (see Schecter \& Montgomery 2004). The numerical integration is started at $r=0$ using the asymptotic behaviour $p(r) \sim r^{|m|}$.

Figure $7(b)$ shows the maximum growth rate of the first branch as a function of $m$ for different values of $\delta$ for $R o=-1$. We see that high azimuthal wavenumbers are stabilized for finite $\delta$, so the most amplified azimuthal wavenumber $m_{\max }$ becomes 
finite. The value of $m_{\max }$ decreases rapidly with $\delta$ (figure $7 b$ ) so that the first branch becomes stable for all $m$ when $\delta>0.038$. A similar stabilization is observed for the second and third branches (figure $7 c$ ). However, the stabilizing effect tends to decrease with the branch number for a given value of $\delta$. Thus, the most amplified wavenumber $m_{\max }$ increases with the branch number, as shown in figure $7(c)$ for $\delta=0.04$.

This stabilization is due to the presence of a singularity at the critical radius $r_{c}$ where $\omega=m U_{\theta}\left(r_{c}\right) / r_{c}$ as soon as $\delta$ is non-zero. As discussed in Schecter \& Montgomery (2004), this singularity has a stabilizing effect whose magnitude is proportional to the vorticity gradient at the critical radius $\left|\zeta^{\prime}\left(r_{c}\right)\right|$. This vorticity gradient can be estimated by using the fact that the critical point is approximately located at $r_{c} \approx R \sqrt{m \Omega / \omega_{r}}$ for small $\delta$. From figure 4, we also see that the frequency of the most unstable mode is $\omega_{r} \approx(m-0.5) \Omega$ independently of $m$. Thus, for large $m$, the vorticity gradient is about $\left|\zeta^{\prime}\left(r_{c}\right)\right| \approx \Omega /\left(R \delta \cosh ^{2}(0.25 /(m \delta))\right)$. This implies that the damping rate is small when $m \delta \ll 1$ and large of order $O(1 / \delta)$ when $m \delta \gg 1$. This explains why the most amplified azimuthal wavenumber scales as $m_{\max }=O(1 / \delta)$ (figure $7 b$ ). The damping rate is smaller for the next branches because the critical radius is located farther from $r=R$ where the vorticity gradient is smaller, since the frequency $\omega_{r}$ of the most amplified mode is slightly smaller than for the first branch.

It seems that this stabilizing effect can render the vortex completely stable or neutral for sufficiently large vorticity smoothness. For example, we have investigated the stability of the Lamb-Oseen vortex whose vorticity profile is very smooth. A Chebyshev spectral stability code (Antkowiak \& Brancher 2007) has been used in order not to miss any unstable mode. Although the growth rates are generally very small, we have always found that they are negative for the range of axial and azimuthal wavenumbers investigated.

\section{Conclusion and discussion}

The stability of the Rankine vortex and the smoothed Rankine vortex has been investigated in a strongly stratified and rotating fluid in the anticyclonic range, $-1 \leqslant R o<0$, which is stable to the centrifugal instability. For the Rankine vortex, the azimuthal wavenumbers $m \geqslant 3$ are unstable, whereas $m=[0,1,2]$ are neutral. The maximum growth rate is $\omega_{i} \approx 0.05 \Omega$, and is reached in the limit of infinite azimuthal wavenumber and for $R o=-1$. The maximum growth rate decreases when the Rossby number is increased from $R o=-1$ and becomes negligible when $R o \gtrsim-0.4$. By means of WKBJ analyses, we have shown that this instability originates from the radiation of inertia-gravity waves. In the case of the smoothed Rankine vortex, a critical layer stabilizes the radiative instability all the more when the vorticity profile is smooth, as reported by Schecter \& Montgomery (2004). In sharp contrast with the Rankine vortex, the most amplified azimuthal wavenumber is finite, and scales in inverse proportion to the smoothness parameter $\delta$.

This radiative instability may operate on anticyclonic geophysical vortices such as the Meddies since the e-folding time $T=1 / \omega_{i}$ can be as low as 10 days if we take a typical turnover time of 2-7 days (Bower, Armi \& Ambar 1997). However, geophysical eddies have a small vertical size, and it would be important to determine whether the radiative instability can operate on such a non-columnar vortex. The stability of other basic flows such as Taylor-Couette flow will also be investigated in the future. 


\section{Acknowledgements}

We thank two anonymous referees for their thoughtful comments. This work was supported by the National Research Agency (ANR 2011 Blanc SIMI 5-6 012-02).

\section{Appendix. WKBJ analysis}

In this appendix, we solve (2.1) for the unstable azimuthal wavenumbers, i.e. $m \geqslant 3$, by means of a WKBJ analysis for large vertical wavenumbers following Billant \& Le Dizès (2009) and Le Dizès \& Billant (2009). As summarized in $\$ 3$, the first condition for the existence of radiative instability is the presence of a region extending towards infinity where the WKBJ approximation (3.1) is wavelike. Thus, we first assume that $\Delta<0$ for $r \rightarrow \infty$. In order to satisfy the boundary condition, we choose that the WKBJ approximation corresponds to an outgoing wave for $r \rightarrow \infty$. Therefore, we set $B=0$ for $r>r_{t}$ in (3.1). In the neighbourhood of the turning point $r_{t}$ where the WKBJ approximation breaks down, (2.1) approximates at leading order to

$$
\frac{\mathrm{d}^{2} p}{\mathrm{~d} \tilde{r}^{2}}-\frac{1}{\tilde{r}} \frac{\mathrm{d} p}{\mathrm{~d} \tilde{r}}+\tilde{r} p=O(\epsilon)
$$

where $\epsilon=1 /\left(-\Delta^{\prime}\left(r_{t}\right) \tilde{k}^{2}\right)^{1 / 3}$ and $\tilde{r}=\left(r-r_{t}\right) / \epsilon$. Note that $\epsilon>0$ since $\Delta^{\prime}\left(r_{t}\right)<0$. The solution of $(\mathrm{A} 1)$ is $p(\tilde{r})=a_{1} \mathrm{Ai}^{\prime}(-\tilde{r})+b_{1} \mathrm{Bi}^{\prime}(-\tilde{r})$, where Ai and Bi denote Airy functions and $a_{1}$ and $b_{1}$ are constants. From the asymptotic behaviour of the Airy functions for $\tilde{r} \rightarrow+\infty$ and $\tilde{r} \rightarrow-\infty$, we find the WKBJ approximation in the region $\left(r<r_{t}\right)$ that matches the outgoing wave for $r>r_{t}$ as

$$
p(r) \sim A \mathrm{e}^{-\mathrm{i} \pi / 4} \frac{\Delta^{1 / 4}}{r^{1 / 2}}\left[\frac{1}{2} \mathrm{e}^{-\tilde{k} \int_{r}^{r_{t}} \sqrt{\Delta(t)} \mathrm{d} t}+\mathrm{ie}^{\tilde{k} \int_{r}^{r_{t}} \sqrt{\Delta(t)} \mathrm{d} t}\right] .
$$

When there is no other turning point between $R$ and $r_{t}$, the approximation (A2) remains valid until $r=R$ and can be directly inserted into the dispersion relation (2.2). This is the case when the frequency is such that $\max \left(\omega_{+}(\infty), \omega_{-}\left(R_{+}\right)\right)<\omega_{r}<\omega_{+}\left(R_{+}\right)$ (see $\S 3$ and figure $1 c$ ). The dispersion relation can be solved explicitly by means of an expansion in powers of $\tilde{k}$. For the first branch for $-2 / 3 \leqslant R o<-2 / m$, the solution (2.3) in the vortex core is always exponential, giving at leading order

$$
\frac{\omega_{r}}{\Omega}=m-\frac{\alpha}{\tilde{k} R \Omega}, \quad \frac{\omega_{i}}{\Omega}=\frac{\alpha^{2}}{2 m} \sqrt{\frac{2}{R o}\left(\frac{2}{R o}+2\right)} \frac{W}{\tilde{k} R \Omega},
$$

where $\quad \alpha=2 m /(|2 / R o|+\sqrt{2(2 / R o+2) / R o})$ and $\quad W=\exp \left(-2 \tilde{k} \int_{R}^{r_{t}} \sqrt{\Delta(t)} \mathrm{d} t\right)$. Equation (A 3) shows that the frequency of the first branch tends to $m \Omega$ as $\tilde{k} \rightarrow \infty$ and its growth rate is of order $O(W / \tilde{k})$. It is in good agreement with the numerical results, as shown by the filled circles in figure 5. Remarkably, the growth rate $\omega_{i}$ is positive even if there is apparently a single wave region. In fact, there is also an infinitely small wave region at $r=R$ due to the vorticity jump of the Rankine vortex. This can be seen by considering the limit $\Delta^{\prime} \gg 1$ in $(2.1)$. When $R o>-2 / m$, the first branch has zero growth rate since there is no turning point, but the frequency $\omega_{r}$ remains identical to (A 3) for large $\tilde{k}$. For the next branches, the solution (2.3) in the vortex core is wavelike, yielding at leading order in $\tilde{k}$

$$
\frac{\omega_{r}}{\Omega}=m+\left(\frac{2}{R o}+2\right)+\frac{\beta^{2}}{2(2 / R o+2)} \frac{1}{(\tilde{k} R \Omega)^{2}},
$$


where $\beta$ satisfies $\beta+\arctan (m / \beta)=\pi(n+(2 m+1) / 4)$ and $n$ is the branch number. In this case, the growth rate is found to be of higher order, $O\left(W / \tilde{k}^{3}\right)$, and is thus much smaller than for the first branch. Equation (A 4) is plotted with open circles in figure 5.

When the frequency is in the range $\omega_{-}(\infty)<\omega_{r}<\omega_{-}\left(R_{+}\right)$, the solution (A 2) is no longer valid at $r=R$ because there is a second turning point (see $\S 3$ and figure $1 c$ ). When $m$ is large, this second turning point $r_{t 2}$ is close to the first one $r_{t 1}$ and also close to the vortex radius $r=R$, so classical WKBJ approximation with two separate turning points (Bender \& Orszag 1978) is not accurate. A better approximation can be obtained by considering the local equation around the radius $r_{o}=R \sqrt{m^{2} /(2 / R o+m \omega / \Omega)}$ where $\Delta$ is maximal (i.e. $\left.\Delta^{\prime}\left(r_{o}\right)=0\right)$ :

$$
\frac{\mathrm{d}^{2} p}{\mathrm{~d} \tilde{r}^{2}}-\frac{2 \tilde{r}}{\tilde{r}^{2}-\lambda} \frac{\mathrm{d} p}{\mathrm{~d} \tilde{r}}+\left(\tilde{r}^{2}-\lambda\right) p=O(\epsilon),
$$

where $\epsilon=R\left(r_{o} / R\right)^{3 / 4} /(2 m \tilde{k} R \Omega)^{1 / 2}, \quad \lambda=\tilde{k} R \Omega\left\{2\left(1+m^{2}\right) / R o^{2}+2 m \omega /(R o \Omega)\right\} /$ $(2 / R o+m \omega / \Omega)^{3 / 2}$ and $\tilde{r}=\left(r-r_{o}\right) / \epsilon$ with $\tilde{r}=O(1)$. The solution of (A 5) is

$p(\tilde{r}) \sim \mathrm{e}^{\mathrm{i} \tilde{r}^{2} / 2}\left[\left(1+\mathrm{i} \tilde{r}^{2}\right) U\left(\frac{\mathrm{i} \lambda+3}{4}, \frac{3}{2},-\mathrm{i} \tilde{r}^{2}\right)+\frac{\mathrm{i}(\mathrm{i} \lambda+3)}{2} \tilde{r}^{2} U\left(\frac{\mathrm{i} \lambda+7}{4}, \frac{5}{2},-\mathrm{i} \tilde{r}^{2}\right)\right]$

where $U$ denotes the Kummer function (Abramowitz \& Stegun 1965). This solution matches the outgoing wave as $\tilde{r} \rightarrow \infty$. When $r_{o}$ is close to $r=R$, (A 6) can be directly inserted into the dispersion relation (2.2) and provides a good prediction for the frequency and the growth rate for large $\tilde{k}$ and $m$, as shown by the dotted lines in figure 4 and crosses in figure 5 .

\section{REFERENCES}

Abramowitz, M. \& Stegun, I. A. 1965 Handbook of Mathematical Functions. Dover.

ANTKOWIAK, A. \& BRANCHER, P. 2007 On vortex rings around vortices: an optimal mechanism. J. Fluid Mech. 578, 295-304.

Bender, C. M. \& Orszag, S. A. 1978 Advanced Mathematical Methods for Scientists and Engineers. McGraw-Hill.

Billant, P. \& Chomaz, J.-M. 2001 Self-similarity of strongly stratified inviscid flows. Phys. Fluids 13, 1645.

Billant, P. \& LE Dizès, S. 2009 Waves on a columnar vortex in a strongly stratified fluid. Phys. Fluids 21, 106602.

Bower, A., ARMi, L. \& AMBAR, I. 1997 Lagrangian observations of Meddy formation during a Mediterranean undercurrent seeding experiment. J. Phys. Oceanogr. 27, 2545-2575.

Broadbent, E. \& Moore, D. W. 1979 Acoustic destabilization of vortices. Phil. Trans. R. Soc. Lond. Ser. A 290, 353.

Candelier, J., Le Dizès, S. \& Millet, C. 2012 Inviscid instability of a stably stratified compressible boundary layer on an inclined surface. J. Fluid Mech. 694, 524-539.

FORD, R. 1994 The instability of an axisymmetric vortex with monotonic potential vorticity in rotating shallow water. J. Fluid Mech. 280, 303-334.

Kloosterziel, R. C. \& van Heijst, G. J. F. 1991 An experimental study of unstable barotropic vortices in a rotating fluid. J. Fluid Mech. 223, 1-24.

LE Dizès, S. \& Billant, P. 2009 Radiative instability in stratified vortices. Phys. Fluids 21, 096602.

LE Dizès, S. \& LACAZE, L. 2005 An asymptotic description of vortex Kelvin modes. J. Fluid Mech. 542, 69-96.

LE Dizès, S. \& RIEDInger, X. 2010 The strato-rotational instability of Taylor-Couette and Keplerian flows. J. Fluid Mech. 660, 147-161. 
Ménesguen, C., Hua, B. L., Papenberg, C., Klaeschen, D., Géli, L. \& Hobbs, R. 2009 Effect of bandwidth on seismic imaging of rotating stratified turbulence surrounding an anticyclonic eddy from field data and numerical simulations. Geophys. Res. Lett. 36, L00D05.

Riedinger, X., Le Dizès, S. \& Meunier, P. 2010 Viscous instability of a Lamb-Oseen vortex in a stratified fluid. J. Fluid Mech. 645, 255-278.

Riedinger, X., Le Dizès, S. \& Meunier, P. 2011 Radiative instability of the flow around a rotating cylinder in a stratified fluid. J. Fluid Mech. 672, 130-146.

SCHECTER, D. A. 2008 The spontaneous imbalance of an atmospheric vortex at high Rossby number. J. Atmos. Sci. 65, 2498.

Schecter, D. A. \& Montgomery, M. T. 2004 Damping and pumping of a vortex Rossby wave in a monotonic cyclone: critical layer stirring versus inertia-buoyancy wave emission. Phys. Fluids 26 (5), 1334.

Schecter, D. A. \& Montgomery, M. T. 2006 Conditions that inhibit the spontaneous radiation of spiral inertia-gravity waves from an intense mesoscale cyclone. J. Atmos. Sci. 63, 435.

SMYth, W. D. \& MCWilliams, J. C. 1998 Instability of an axisymmetric vortex in a stably stratified, rotating environment. Theor. Comput. Fluid Dyn. 11, 305-322.

VANNESTE, J. \& YAVNEH, I. 2004 Exponentially small inertia-gravity waves and the breakdown of quasigeostrophic balance. J. Atmos. Sci. 61, 211-223.

VANNESTE, J. \& YAVNEH, I. 2007 Unbalanced instabilities of rapidly rotating stratified shear flows. J. Fluid Mech. 584, 373-396. 